\title{
Infección del sitio quirúrgico debido al uso de instrumental de laparoscopia tratado mediante desinfección de alto nivel con glutaraldehído al $2 \%$ : revisión sistemática
}

\author{
Surgical site infection due to use of laparoscopic instruments treated \\ by high-level disinfection with $2 \%$ glutaraldehyde: A systematic review
}

\author{
Judy A. Ruíz-Ochoa IQ1, \\ Christopher S. Valdés-Cataño IQ², Viviana Arcila-Olmos $\mathrm{MSc}^{3}$
}

Introducción: las infecciones del sitio quirúrgico representan el $25 \%$ de las infecciones asociadas al cuidado de la salud, por lo que el procesamiento del instrumental de laparoscopia es de gran relevancia. La inmersión de este material en glutaraldehído al 2\% para eliminar microorganismos ha demostrado ser costoefectiva. Objetivo: describir información sobre el riesgo de adquirir infecciones del sitio quirúrgico tras el uso de instrumental de cirugía laparoscópica tratado con soluciones de glutaraldehído al 2\%. Materiales y métodos: se realizó una búsqueda sistemática en las bases de datos LILACS, SciELO, JSTOR, OVID-Medline, PubMed-Medline, Scopus, Google Scholar y la Colaboración Cochrane de artículos publicados entre 1980 y 2013, que trataran sobre infecciones del sitio quirúrgico debido a instrumental laparoscópico procesado con glutaraldehído al 2\%. Resultados: se seleccionaron nueve artículos, publicados en la India, Turquía, México y Brasil, seis referentes a casos de infección del sitio quirúrgico en pacientes sometidos a laparoscopia con material desinfectado con glutaraldehído al 2\%, dos al aislamiento de microorganismos a partir de trócares laparoscópicos después de ha-

\footnotetext{
1 Instrumentadora quirúrgica, Programa de Instrumentación Quirúrgica, Facultad de Medicina, Universidad de Antioquia. Medellín, Colombia. Correspondencia: Calle 32D \# 65F 87. Correo electrónico: judy58397@hotmail.com

${ }^{2}$ Instrumentador quirúrgico, Programa de Instrumentación Quirúrgica, Universidad de Antioquia. Medellín, Colombia.

${ }^{3}$ Instrumentadora quirúrgica, Especialista en Salud Ocupacional, MSc en Epidemiología. Docente Programa de Instrumentación Quirúrgica, Facultad de Medicina, Universidad de Antioquia. Medellín, Colombia.

Conflicto de intereses: los autores declaran que no tienen conflictos de intereses Medicina \& Laboratorio 2016; 22: 55-70

Módulo 7 (Microbiología), número 28. Editora Médica Colombiana S.A. $2016^{\odot}$

Recibido el 27 de abril de 2014; aceptado el 01 de febrero de 2016
}

Medicina \& Laboratorio / Volumen 22, Números 1-2, 2016. 
ber sido desinfectados con glutaraldehído al 2\%, y uno a ambas evaluaciones. Conclusiones: las condiciones no óptimas de almacenamiento, preparación, limpieza y dilución del glutaraldehído al 2\%, sumado al personal sin la debida capacitación para su manejo y la implicación de microorganismos atípicos como Mycobacterium masiliense como agentes causales de infección del sitio quirúrgico, están asociadas a la falta de efectividad del proceso de desinfección de alto nivel del instrumental laparoscópico, que lleva a reevaluar su uso en las instituciones de salud.

Palabras clave: desinfección, laparoscopía, glutaral, infección hospitalaria.

Abstract: Surgical site infections account for $25 \%$ of care-associated infections health, so the laparoscopic instrument processing is highly relevant. Immersion of this material in 2\% glutaraldehyde to eliminate microorganisms has proven to be cost-effective. Objective: To describe information about the risk of acquiring surgical site infections after the use of laparoscopic surgery instruments treated with 2\% glutaraldehyde solutions. Materials and methods: A systematic search on databases LILACS, SciELO, JSTOR, OVID-Medline, PubMed-Medline, Scopus, Google Scholar and Cochrane Collaboration of articles published between 1980 and 2013 about surgical site infections due to use of surgical laparoscopic instruments processed with $2 \%$ glutaraldehyde. Results: Nine articles published in India, Turkey, Mexico, and Brazil were selected, six of them concerning of cases of surgical site infection in patients undergoing laparoscopy with material disinfected with $2 \%$ glutaraldehyde, two about microorganisms isolation from laparoscopic trocars disinfected with $2 \%$ glutaraldehyde and the other one about both evaluations. Conclusions: non-optimal storage conditions, preparation, cleaning and dilution of $2 \%$ glutaraldehyde, added to personnel without proper training for management and involvement of atypical microorganisms such as Mycobacterium masiliense as causative agents of surgical site infection, are associated with the lack of effectiveness of the high-level disinfection process of laparoscopic instruments, leading to reevaluate its use in health institutions.

Keywords: disinfection, laparoscopy, glutaral, cross infection.

Ruíz-Ochoa JA, Valdés-Cataño CS, Arcila-Olmos V. Infección del sitio quirúrgico debido al uso de instrumental de laparoscopia tratado mediante desinfección de alto nivel con glutaraldehído al 2\%: revisión sistemática. Medicina \& Laboratorio 2016; 22: 55-70.

$\mathrm{E}^{\mathrm{n}}$ n las últimas décadas se han realizado diversos intentos por llevar a cabo procedimientos quirúrgicos con la menor irrupción posible en el cuerpo del paciente, los cuales han permitido el desarrollo de técnicas de cirugía mínimamente invasivas $[1,2]$ aceptadas, actualmente, por parte del personal del área de la salud y de los pacientes como primera opción quirúrgica [2, 3]. Estos procedimientos han demostrado ciertas ventajas respecto a los métodos convencionales, 
entre los que se incluyen la disminución de los tiempos quirúrgicos y la estancia hospitalaria $[1,2,4]$, la reducción de complicaciones posoperatorias como hernias y adherencias gracias a la disminución en la manipulación de los tejidos [4] y el impacto estéticamente favorable al obtener cicatrices de menor tamaño [5], lo que a su vez lleva al retorno más rápido del paciente a su vida habitual [4].

Una de las preocupaciones más importantes en cualquier procedimiento quirúrgico es la posible introducción de microorganismos a los sitios estériles del cuerpo que aumenten el riesgo de sufrir una infección $[6,7]$. Específicamente, la infección del sitio quirúrgico representa el $25 \%$ de las infecciones asociadas al cuidado de la salud [8], en las cuales los equipos e instrumental que se utilizan durante una cirugía son unos de los componentes fundamentales de la cadena de transmisión de estas infecciones [8,9]. Esta situación ha vuelto indispensable realizar un adecuado procesamiento del instrumental que se utiliza durante las cirugías para reducir al mínimo la carga microbiana y, por ende, el riesgo de que ocurra una infección.

El instrumental específico para las cirugías abiertas, al presentar de alguna manera un diseño más sencillo, permite con facilidad su limpieza y esterilización mediante métodos convencionales como el vapor de agua a presión, el cual inyecta un vapor de agua entre $121{ }^{\circ} \mathrm{C}$ y $134{ }^{\circ} \mathrm{C}$ en un tiempo constante. Este método, debido a su eficacia, capacidad de penetración y distribución equitativa del vapor en los dispositivos médicos utilizados, su fiabilidad, facilidad de monitorización, seguridad (debido a la ausencia de residuos tóxicos) y ser económico, es el más utilizado en los servicios hospitalarios [10-14].

Por otro lado, la limpieza y esterilización del instrumental para cirugía mínimamente invasiva es más compleja, debido a que presenta diseños más complicados desde el punto de vista mecánico, algunos de los cuales no son desmontables y poseen espacios internos de difícil acceso, lo que impide la reducción de la materia orgánica durante el lavado $[15,16]$. Además, los instrumentos para laparoscopia son fabricados con elementos termosensibles que pueden sufrir dilataciones y otros daños en su estructura cuando son sometidos a altas temperaturas como las que se alcanzan mediante el sistema de esterilización con vapor de agua a presión [15].

El autoclave, al ser un método de esterilización con vapor de agua a presión, deteriora y causa daño a los dispositivos termosensibles [13], lo que obliga a que sean procesados mediante otro tipo de métodos como el óxido de etileno, que es un gas con alta capacidad de penetración que se encuentra en el mercado en estado puro o mezclado con freón o dióxido de carbono y que logra la eliminación de microorganismos mediante la alquilación de proteínas estructurales, enzimas y ácidos nucleicos a una temperatura ente $30^{\circ} \mathrm{C}$ y $60^{\circ} \mathrm{C}$. En comparación con el método de vapor de agua a presión la esterilización con óxido de etileno es cos- 
tosa y requiere más tiempo, puesto que por su toxicidad los dispositivos luego de ser sometidos al proceso deben contar con un tiempo de aireación estandarizado para lograr así la eliminación del gas en el instrumental expuesto $[10,17,18]$, lo que lleva a una inactividad del mismo y a un cese de la productividad del equipo.

Otro de los métodos utilizados para la esterilización del material quirúrgico es la radiación gamma proveniente de fuentes como el Cobalto-60, cuyo efecto se debe a la creación de radicales en los componentes celulares, lo que inhibe la propagación celular y lleva finalmente a la muerte del microorganismo. Este método esteriliza dispositivos sin importar el espesor del material, el producto y el tipo de empaque, y no requiere de tiempo de aireación pues presenta un bajo nivel de toxicidad, reacción química, nivel de residuos y mínimo cambio en su temperatura; no obstante, su principal inconveniente es el alto costo del equipo y de la instalación, ya que debe cumplir con altas medidas de seguridad [19, 20].

El método de esterilización con plasma de peróxido de hidrógeno consiste en la transformación de una solución acuosa de esta sustancia a una concentración de $58 \%$ en una nube de iones reactivos, electrones y partículas atómicas neutras con capacidad de esterilizar, empleando temperaturas por debajo de $50{ }^{\circ} \mathrm{C}$, mediante un sinergismo entre la acción oxidante del peróxido de hidrógeno y la actividad alquilante de los radicales libres. Los residuos finales de este proceso son oxígeno y agua. La principal desventaja de este método es que los materiales a esterilizar mediante el mismo deben poseer unas características particulares tales como la ausencia de lúmenes largos debido a que la capacidad de difusión del peróxido de hidrógeno es baja. Tampoco es posible esterilizar cargas que contengan materiales basados en celulosa como madera, algodón o papel; razón por la que los empaques para esterilizar con este método están fabricados a partir de materias primas libres de fibras naturales [10, 21-23]. Finalmente, el vapor de formaldehído es el método de esterilización menos usado en América, además es más tóxico que el óxido de etileno y no se ha comprobado totalmente su eficacia para la esterilización $[10,15]$.

Teniendo en cuenta que el procesamiento de dispositivos médicos mediante técnicas de baja temperatura supone costos más altos a causa de la tecnología que emplean, se ha optado por buscar alternativas que proporcionen tanto efectividad como rapidez en el procesamiento de los dispositivos médicos, así como un menor costo en comparación con otros métodos de esterilización. Las instituciones de salud se han inclinado por la utilización de la inmersión en sustancias químicas para desinfectar el instrumental.

La desinfección corresponde al proceso con el cual se logra eliminar todos los microorganismos presentes en una superficie inanimada, con excepción de las formas esporuladas de los mismos [6, 23]. Dado que no todos los desinfectantes tienen el mismo efecto microbicida sobre todos los tipos de microorganismos se 
diseñó un sistema de clasificación en tres niveles que permitiera establecer los parámetros para el uso adecuado de las diferentes sustancias según las necesidades específicas.

La desinfección de bajo nivel corresponde a un proceso químico con capacidad para eliminar las formas vegetativas de las bacterias, así como virus y hongos, pero no elimina a Mycobacterium tuberculosis ni las esporas bacterianas. Los amonios cuaternarios son los desinfectantes de bajo nivel más ampliamente difundidos. La principal diferencia entre la desinfección de bajo nivel y el proceso de desinfección de nivel intermedio, logrado mediante la aplicación de sustancias como el alcohol etílico e isopropílico, el hipoclorito de sodio, los fenoles, la cetrimida y el cloruro de benzalconio, radica en la capacidad de este último para eliminar el complejo Mycobacterium tuberculosis. Por último, con la desinfección de alto nivel, teóricamente, es posible eliminar toda clase de virus, hongos y formas vegetativas de bacterias, incluyendo las micobacterias. A pesar de que la diferencia entre el proceso de esterilización y la desinfección de alto nivel es la inhabilidad de esta última para destruir las esporas bacterianas se ha descrito que con sustancias como el glutaraldehído y el ortoftalaldehído es posible lograrlo después de tiempos de contacto tan prolongados como 12 horas $[6,22,24]$.

Earle H. Spaulding, médico y profesor emérito del Departamento de Microbiología de la Facultad de Medicina de la Temple University, hace más de treinta años, clasificó los dispositivos médicos en tres categorías, críticos, semicríticos y no críticos, de acuerdo al riesgo de infección que corre el paciente con el uso de los mismos. Un dispositivo se considera crítico cuando entra en contacto con tejidos y cavidades estériles del organismo (p. ej. los instrumentos quirúrgicos), así como con el sistema vascular (p. ej. catéteres y stents); razón por la que deben ser esterilizados antes de su utilización $[6,25,26]$. Los elementos que entran en contacto con membranas mucosas y piel no intacta son incluidos en la categoría de semicríticos y antes de ser usados se recomienda que, como mínimo, sean sometidos a un proceso de desinfección de alto nivel, puesto que se ha demostrado que las membranas mucosas de los tractos respiratorio y gastrointestinal son resistentes a las infecciones por esporas bacterianas [10]. Finalmente, los elementos no críticos, considerados así por tratarse de aquellos que únicamente tienen contacto con la piel indemne del paciente, la cual representa una barrera efectiva contra la mayoría de los microorganismos, solo requieren de un procedimiento de desinfección de nivel bajo o intermedio antes y después de cada uso $[25,26]$.

De lo anterior es posible deducir que el instrumental laparoscópico es un elemento crítico y, por lo tanto, se debe usar siempre estéril; sin embargo, dado que el procesamiento de los dispositivos médicos mediante técnicas de baja temperatura supone costos más altos debido a la tecnología empleada, varias instituciones alrededor del mundo han optado por buscar alternativas de tratamientos que 
proporcionen tanto efectividad como rapidez en el reprocesamiento de los dispositivos médicos, así como un menor costo en comparación con otros métodos de esterilización. En este sentido, la inmersión del instrumental médico en sustancias químicas como el glutaraldehído al $2 \%$ ha resultado ser una alternativa útil para cubrir estos requerimientos $[24,27]$.

La eficacia del uso de sustancias químicas como desinfectantes de alto nivel depende de diversos factores tanto intrínsecos como extrínsecos, como el entrenamiento adecuado del personal encargado de su manipulación, las condiciones en las que se deben almacenar, la concentración del agente y la cantidad de materia orgánica presente en las superficies de los dispositivos, encargada de proteger a los microorganismos, que puede inactivar el producto $[26,28]$. Este último factor, a su vez, modula el tiempo requerido para llevar a cabo un buen proceso de desinfección de alto nivel [27].

El tiempo transcurrido desde la preparación de la solución desinfectante hasta su uso también influye. Igualmente, se debe considerar el tipo de microorganismos presentes en los restos de material que quedan en un dispositivo tras su uso, debido a que, dependiendo de su naturaleza, hay microorganismos que pueden ser resistentes a estas sustancias por lo requieren ser sometidos a mayores períodos para ser destruidos [26, 28-32]. Además, la modificación de los protocolos de preparación de las soluciones de desinfección de alto nivel por personal inexperto supone un riesgo potencial para el paciente si se tiene en cuenta que el instrumental, finalmente, va a tener contacto con un sitio estéril del organismo [25, 26, $30,31,33]$.

Dentro de esta problemática se ha de sumar que el aumento de la estancia hospitalaria secundaria a las infecciones del sitio quirúrgico y la utilización de antibióticos de amplio espectro para controlarlas no son costo-efectivamente viables para ninguna institución y mucho menos para el paciente, por lo que se convierten en una carga económica para el presupuesto institucional [34-36].

Teniendo en cuenta lo anterior, se hace importante identificar si existe riesgo de adquisición de infecciones del sitio quirúrgico tras el uso de instrumental de cirugía laparoscópica inmerso en soluciones de glutaraldehído al 2\% para su desinfección, sirviendo esta revisión como guía para su uso en las instituciones de salud. En el presente artículo se reportan los hallazgos de una revisión bibliográfica sistemática sobre la infección del sitio quirúrgico debido al uso de instrumental de laparoscopia tratado mediante desinfección de alto nivel con glutaraldehído al $2 \%$.

\section{Materiales y métodos}

Se realizó una revisión sistemática de la literatura médica sobre las infecciones del sitio quirúrgico en pacientes en los cuales el instrumental de cirugía no fue este- 
rilizado, sino procesado mediante desinfección de alto nivel con glutaraldehído al $2 \%$. La búsqueda se realizó en las bases de datos científicas LILACS (Literatura Latinoamericana y del Caribe en Ciencias de la Salud), PubMed-Medline, OVIDMedLine, JSTOR (Journal Storage), SciELO (Scientific Electronic Library Online), Scopus, Google Scholar y la Colaboración Cochrane entre los meses de febrero y octubre de 2013, utilizando los términos: «high level disinfection AND laparoscopic» («desinfección de alto nivel Y laparoscopia»). Además, se analizó la información procedente de las páginas web de diversas sociedades científicas y organizaciones que desarrollan temas asociados al contenido que se pretendía sintetizar en esta revisión sistemática.

Se seleccionaron los artículos tipo carta al editor, reporte de casos, ensayo clínico controlado aleatorizado y estudio de investigación publicados entre 1980 y 2013, en los idiomas español, inglés y portugués, que hacían referencia a pacientes infectados tras una intervención de cirugía laparoscópica con uso de instrumental tratado mediante desinfección de alto nivel, o a la cantidad de instrumentos contaminados tras este mismo tratamiento. Se excluyeron los artículos no disponibles en versión electrónica y aquellos con fecha de publicación anterior a 1980. Los artículos fueron examinados de acuerdo al título y su resumen. Se tuvieron en cuenta aquellos que cumplían con los criterios de inclusión y los aspectos relacionados con el objetivo de este estudio sistemático como tipo de cirugía, instrumental utilizado y agentes etiológicos causantes de infección.

\section{Resultados}

La búsqueda de los términos en las bases de datos seleccionadas arrojó un total de 148 artículos de los cuales 100 se obtuvieron en la base de datos OVID-Medline, 31 de JSTOR, nueve de PubMed-Medline, siete de Scopus y uno mediante Google Scholar. No se obtuvieron artículos compatibles con los criterios de búsqueda en la biblioteca de la Colaboración Cochrane ni en las bases de datos de SciELO y LILACS. Se llevó a cabo la lectura de los resúmenes de estos artículos y 31 fueron seleccionados para su lectura completa teniendo en cuenta el cumplimiento de los criterios de inclusión mencionados.

Se descartaron los artículos que estaban en idiomas diferentes al inglés, español y portugués, los que no fueron realizados en humanos y los que no incluían la desinfección de alto nivel, la infección, la laparoscopia y los instrumentos contaminados tras el tratamiento por glutaraldehído al $2 \%$. Nueve de los artículos cumplieron con los criterios de inclusión para esta revisión (véase figura 1 y tabla 1).

Los países de donde procedieron los nueve estudios fueron principalmente India y Turquía con tres publicaciones cada uno. De Brasil se encontraron dos estudios enfocados en un mismo evento epidemiológico ocurrido entre agosto de 2006 y 
julio de 2007 en este país, los cuales complementaban sus resultados. Por último, se encontró un artículo de procedencia mexicana. El período de publicación estuvo comprendido entre 1998 y 2009 (véase tabla 1).

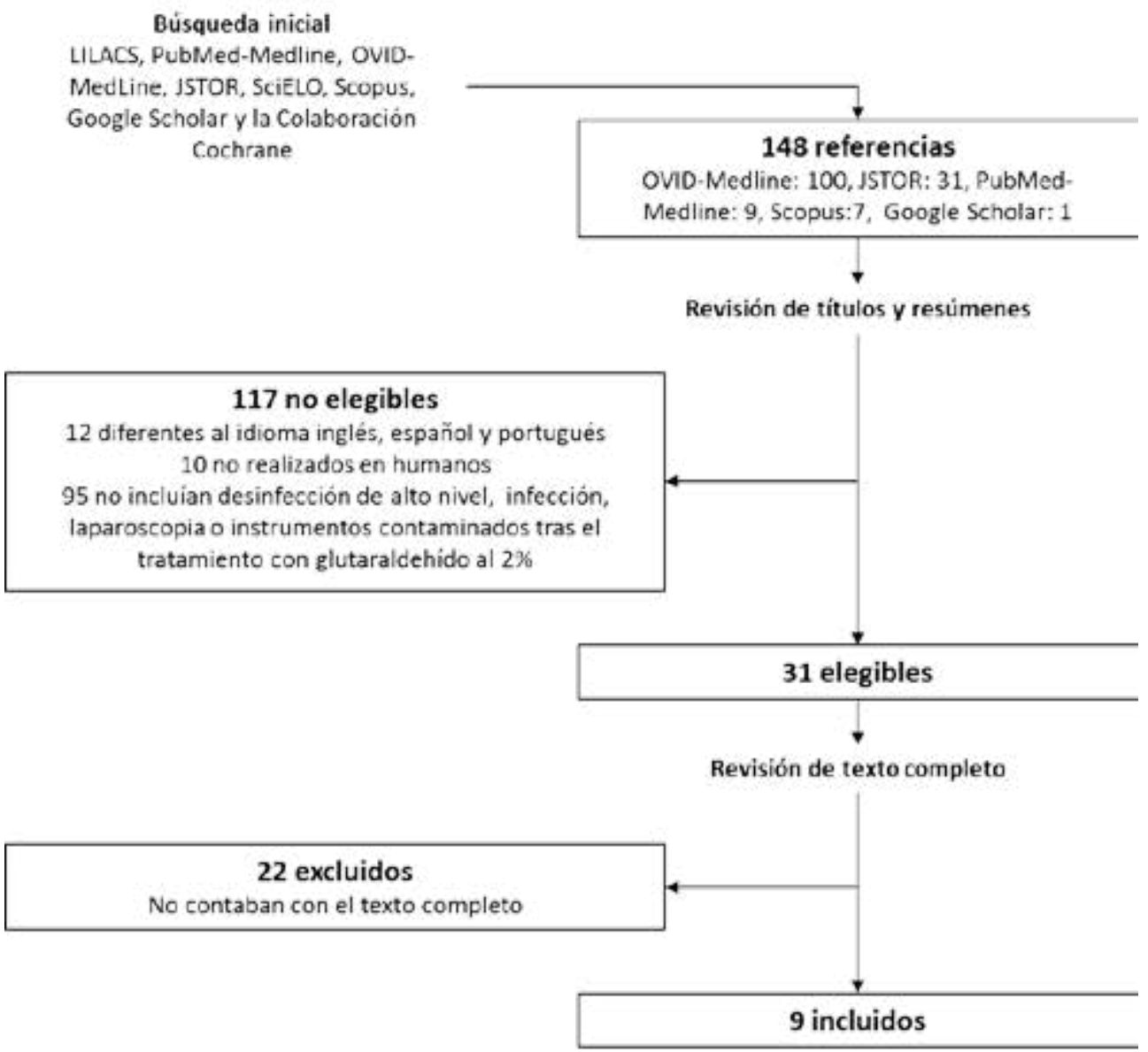

Figura 1. Esquema de la revisión sistemática de los estudios sobre infección del sitio quirúrgico debido al uso de instrumental de laparoscopia tratado mediante desinfección de alto nivel con glutaraldehído al $2 \%$.

El consolidado del número de pacientes con procedimientos quirúrgicos documentados en siete de las nueve publicaciones seleccionadas arrojó un total de 1.360 casos. Dos de los estudios estuvieron enfocados únicamente en el procedimiento de colecistectomía laparoscópica [37, 40], mientras que en los otros cinco (contando ambas publicaciones de Brasil), la laparoscopia estuvo incluida con otros tipos de cirugía $[38,42,43]$. El consolidado de los procedimientos quirúrgicos más frecuentes indicó que la colecistectomía laparoscópica fue la más utilizada, correspondiente al $52,06 \%$ del total de procedimientos realizados. Entre las demás indicaciones se encontraron: laparoscopias diagnósticas $(6,47 \%)$, apendicectomía (5,51\%), artroscopia $(4,26 \%)$, adhesiolisis $(0,51 \%)$, miomectomía $(1,84 \%)$, ooforectomía $(2,50 \%)$, 


\begin{abstract}
Tabla 1. Estudios incluidos en la revisión bibliográfica sistemática sobre infección del sitio quirúrgico debido al uso de instrumental de laparoscopia tratado mediante desinfección de alto nivel con glutaraldehído al $\mathbf{2 \%}$
\end{abstract}

\begin{tabular}{|c|c|c|c|c|c|}
\hline Autor & Año & $\begin{array}{l}\text { Lugar del } \\
\text { estudio }\end{array}$ & Tipo de estudio & $\begin{array}{l}\text { Período de } \\
\text { estudio }\end{array}$ & Muestra y principales hallazgos \\
\hline Zayyan & 1998 & Turquía & Carta & No & - 15 hombres y 30 mujeres some \\
\hline
\end{tabular}

Zayyan 1998 Turquía Carta No Nos $\quad$-15 hombres y 30 mujeres sometidos

\& Sellu [37] al editor registrado a colecistectomía laparoscópica. En

30 de ellos se usó material tratado con desinfección de alto nivel.

-15 trócares laparoscópicos para evaluación de contaminación después de ser desinfectados con glutaraldehído al $2 \%$

$\begin{array}{lllll}\begin{array}{l}\text { Rodrigues y } \\ \text { colaboradores } \\ \text { [38] }\end{array} & 2001 & \text { India } & \text { Carta al editor } & \begin{array}{l}\text { No } \\ \text { registrado }\end{array} \\ & & & & \\ & & & & \\ \begin{array}{l}\text { Ramesh y } \\ \text { colaboradores }\end{array} & 2003 & \text { India } & \begin{array}{l}\text { Reporte de } \\ \text { casos }\end{array} & \begin{array}{l}1996 a \\ 2000\end{array}\end{array}$

33 pacientes sometidos a cirugía laparoscópica con instrumentos sometidos a desinfección de alto nivel y 16 reingresos por infección del sitio quirúrgico, 13 de ellos con cultivos positivos para Mycobacterium chelonae

colaboradores [39]

\begin{tabular}{lllll}
$\begin{array}{l}\text { Colak } \\
\text { y colaboradores } \\
\text { [40] }\end{array}$ & 2004 & Turquía & $\begin{array}{l}\text { Ensayo clínico } \\
\text { controlado } \\
\text { aleatorizado }\end{array}$ & $\begin{array}{l}\text { Enero a } \\
\text { agosto de } \\
2002\end{array}$ \\
$\begin{array}{l}\text { Vijayaraghav } \\
\begin{array}{l}\text { y colaborado- } \\
\text { res [41] }\end{array}\end{array}$ & & India & $\begin{array}{l}\text { Estudio de } \\
\text { investigación }\end{array}$ & $\begin{array}{l}\text { Diciembre } \\
\text { de } 2002 \\
\text { a junio de } \\
2003\end{array}$ \\
\hline
\end{tabular}

\begin{tabular}{|c|c|c|c|c|c|}
\hline $\begin{array}{l}\text { Lorena, Duarte } \\
\text { \& Pitombo } \\
\text { [42] } \\
\text { Duarte } \\
\text { y colaboradores } \\
\text { [43] }\end{array}$ & 2009 & Brasil & $\begin{array}{l}\text { Estudio de } \\
\text { investigación }\end{array}$ & $\begin{array}{l}\text { Agosto } \\
\text { de } 2006 \\
\text { a julio de } \\
2007\end{array}$ & $\begin{array}{l}1.051 \text { posibles casos de infección } \\
\text { del sitio quirúrgico asociada a ins- } \\
\text { trumentos laparoscópicos sometidos } \\
\text { a desinfección con glutaraldehído al } \\
2 \% \text {, atendidos en } 63 \text { hospitales: De } \\
\text { ellos, } 302 \text { casos fueron confirmados } \\
\text { por el laboratorio, de los cuales } 172 \\
\text { presentaron un cultivo positivo para } \\
\text { micobacterias atípicas. De los } 148 \\
\text { aislamientos disponibles } 144 \text { fueron } \\
\text { identificados como Mycobacterium } \\
\text { masiliense }\end{array}$ \\
\hline $\begin{array}{l}\text { Ulualp } \\
\text { y colaboradores } \\
{[44]}\end{array}$ & 2000 & Turquía & $\begin{array}{l}\text { Ensayo clínico } \\
\text { controlado } \\
\text { aleatorizado }\end{array}$ & 2000 & $\begin{array}{l}\text { Evaluación de contaminación en } 40 \\
\text { trócares laparoscópicos después de } \\
\text { ser desinfectados con glutaraldehído } \\
\text { al } 2 \%\end{array}$ \\
\hline $\begin{array}{l}\text { Villagrana y } \\
\text { colaboradores } \\
{[45]}\end{array}$ & 2002 & México & $\begin{array}{l}\text { Estudio de } \\
\text { investigación }\end{array}$ & $\begin{array}{l}\text { Marzo a } \\
\text { octubre de } \\
1999\end{array}$ & $\begin{array}{l}\text { Evaluación de contaminación en } 416 \\
\text { trócares laparoscópicos s después de } \\
\text { ser desinfectados con glutaraldehído } \\
\text { al } 2 \%\end{array}$ \\
\hline
\end{tabular}

12 pacientes con infección del sitio quirúrgico. Ocho de ellos, seis mujeres y dos hombres sometidos a cirugía laparoscópica, y con evidencia histológica de tuberculosis. Tres presentaron cultivo microbiológico positivo para Mycobacterium tuberculosis

153 pacientes sometidos a colecistectomía laparoscópica. En 63 de ellos se usó instrumental tratado con glutaraldehído al $2 \%$, de los cuales dos presentaron infección del sitio quirúrgico

156 pacientes sometidos a cirugía laparoscópica con material procesado con glutaraldehído al $2 \%$, atendidos en un período de seis meses. En 35 de ellos, 16 hombres y 19 mujeres, se obtuvo cultivo positivo en la herida quirúrgica para micobacterias atípicas

1.051 posibles casos de infección a desinfección con glutaraldehído al $2 \%$, atendidos en 63 hospitales: De ellos, 302 casos fueron confirmados micobacterias atípicas. De los 148 Evaluación de contaminación en 40 ser desinfectados con glutaraldehído 
tubectomía $(1,99 \%)$, hernipolastia $(1,84 \%)$, cistectomía $(0,37 \%)$, gastroplastia $(1,18 \%)$, histerectomía $(0,29 \%)$, rectosigmoidectomía $(1,18 \%)$ y tratamiento quirúrgico de endometriosis (0,07\%). No obstante, en el $19,93 \%$ de los casos el procedimiento laparoscópico no fue especificado (véase tabla 2).

Uno de los objetos de investigación de seis de las siete publicaciones fueron las infecciones del sitio quirúrgico [37-43]. El consolidado de casos de infección del sitio quirúrgico fue de 367 , correspondiente al $26,99 \%$ del total de procedimientos quirúrgicos documentados. En estos casos se recolectaron muestras de tejido y de secreciones en las heridas que fueron sometidas a pruebas de laboratorio como tinción de Gram, tinción de Ziehl-Nielsen y otras especificadas en cada estudio. Además, se realizó el aislamiento de los agentes etiológicos mediante cultivo, el cual se encontró positivo en un consolidado de 225 pacientes, correspondiente al $61,31 \%$ de los casos con infección del sitio quirúrgico y al $16,54 \%$ del total de procedimientos realizados.

El microrganismo implicado en la infección del sitio quirúrgico varió en función de la ubicación geográfica de cada estudio. Dos de las publicaciones originarias de la India encontraron como agente causal a Mycobacterium chelonae $[38,41]$, mientras que en un tercer estudio en este mismo país se encontró a Mycobacterium tuberculosis como el agente etiológico [39]. Entretanto, los dos estudios brasileros confirmaron la presencia de una micobacteria atípica, Mycobacterium masiliense, en 144 de los 148 aislamientos disponibles para el análisis $[42,43]$. Finalmente, uno de los estudios de Turquía encontró implicados microorganismos diferentes a micobacterias; sin embargo, este resultado se atribuye a la contaminación directa con la microbiota de la piel del mismo paciente más que al proceso de desinfección, dado que no se obtuvo crecimiento bacteriano en las muestras tomadas de los trócares laparoscópicos reutilizados [40] (véase tabla 2).

La presencia de microorganismos en el instrumental quirúrgico fue evaluada en tres de los nueve estudios [37, 44, 45], que en total analizaron 471 trócares de laparoscopia después de haber sido sumergidos en soluciones similares de glutaraldehído al $2 \%$ durante un tiempo promedio de 20 minutos, siguiendo los protocolos adecuados de limpieza y manipulación por personal calificado y bajo las condiciones adecuadas para tal procedimiento. A pesar de estas medidas se evidenció la presencia de diferentes microorganismos en ocho $(1,70 \%)$ del total de trócares. Los microorganismos aislados correspondieron a las bacterias Bacillus stearothermophilus, Pseudomonas aeruginosa, Enterococcus spp. y otros bacilos Gram positivos no especificados y el hongo Aspergillus spp. (véase tabla 3). 


\begin{tabular}{|c|c|c|c|c|c|c|c|}
\hline $\begin{array}{l}\text { Autor } \\
\text { (referencia) }\end{array}$ & $\begin{array}{l}\text { Procedimientos } \\
\text { quirúrgicos }\end{array}$ & $\begin{array}{l}\mathrm{N} .^{\circ} \text { de } \\
\text { pacientes } \\
\text { por proce- } \\
\text { dimiento } \\
\text { quirúrgico }\end{array}$ & $\begin{array}{l}\mathrm{N}^{\circ} \\
\text { total de } \\
\text { pacientes } \\
\text { estudiados }\end{array}$ & $\begin{array}{l}\text { N. }{ }^{\circ} \text { de } \\
\text { pacien- } \\
\text { tes con } \\
\text { infección } \\
\text { del sitio } \\
\text { quirúrgico }\end{array}$ & $\begin{array}{l}\text { N. }{ }^{\circ} \text { de } \\
\text { pacien- } \\
\text { tes con } \\
\text { cultivo } \\
\text { positivo }\end{array}$ & $\begin{array}{l}\text { Principales } \\
\text { microor- } \\
\text { ganismos } \\
\text { aislados en } \\
\text { la herida } \\
\text { quirúrgica }\end{array}$ & $\begin{array}{l}\text { Tiempo de } \\
\text { inmersión } \\
\text { del instru- } \\
\text { mental en } \\
\text { glutaralde- } \\
\text { hído al 2\% }\end{array}$ \\
\hline $\begin{array}{l}\text { Zayyan \& } \\
\text { Sellu [37] }\end{array}$ & Colecistectomía & 45 & 45 & 0 & No aplica & No aplica & $\begin{array}{l}\text { No } \\
\text { especificado }\end{array}$ \\
\hline \multirow{7}{*}{$\begin{array}{l}\text { Rodrigues } \\
\text { y colaboradores } \\
\text { [38] }\end{array}$} & Colecistectomía & 11 & \multirow[t]{7}{*}{33} & \multirow[t]{7}{*}{16} & \multirow[t]{7}{*}{13} & \multirow{7}{*}{$\begin{array}{l}\text { Mycobac- } \\
\text { terium } \\
\text { chelonae }\end{array}$} & \multirow{7}{*}{$\begin{array}{l}\text { No } \\
\text { especificado }\end{array}$} \\
\hline & $\begin{array}{l}\text { Laparoscopia } \\
\text { diagnóstica }\end{array}$ & 6 & & & & & \\
\hline & Adhesiolisis & 5 & & & & & \\
\hline & Cistectomía & 5 & & & & & \\
\hline & Tubectomía & 3 & & & & & \\
\hline & $\begin{array}{l}\text { Histerectomía } \\
\text { vaginal asistida } \\
\text { por laparoscopia }\end{array}$ & 2 & & & & & \\
\hline & Miomectomía & 1 & & & & & \\
\hline \multirow{7}{*}{$\begin{array}{l}\text { Ramesh } \\
\text { y colaboradores } \\
\text { [39] }\end{array}$} & Colecistectomía & 1 & \multirow[t]{7}{*}{12} & \multirow{7}{*}{$\begin{array}{l}12 \text { (8 con } \\
\text { evidencia } \\
\text { histológica } \\
\text { de } \\
\text { tuberculosis) }\end{array}$} & \multirow[t]{7}{*}{3} & \multirow{7}{*}{$\begin{array}{l}\text { Mycobac- } \\
\text { terium } \\
\text { tubercu- } \\
\text { losis }\end{array}$} & \multirow[t]{7}{*}{20 minutos } \\
\hline & Adhesiolisis & 2 & & & & & \\
\hline & Histerectomía & 2 & & & & & \\
\hline & $\begin{array}{l}\text { Tratamiento de } \\
\text { endometriosis }\end{array}$ & 1 & & & & & \\
\hline & Ooferectomía & 1 & & & & & \\
\hline & Hernioplastia & 1 & & & & & \\
\hline & $\begin{array}{l}\text { Cirugía laparoscópi- } \\
\text { ca no especificada }\end{array}$ & 4 & & & & & \\
\hline $\begin{array}{l}\text { Colak } \\
\text { y colaboradores } \\
{[40]}\end{array}$ & Colecistectomía & 63 & 63 & 2 & 2 & $\begin{array}{l}\text { Staphylo- } \\
\text { coccus } \\
\text { aureus y } \\
\text { Esche- } \\
\text { richia coli }\end{array}$ & 20 minutos \\
\hline $\begin{array}{l}\text { Vijayaraghav y } \\
\text { colaboradores } \\
{[41]}\end{array}$ & $\begin{array}{l}\text { Cirugía laparoscópi- } \\
\text { ca no especificada }\end{array}$ & 156 & 156 & 35 & 35 & $\begin{array}{l}\text { Mycobac- } \\
\text { terium } \\
\text { chelonae }\end{array}$ & 60 minutos \\
\hline \multirow{3}{*}{$\begin{array}{l}\text { Lorena, } \\
\text { Duarte \& } \\
\text { Pitombo } \\
{[42]}\end{array}$} & Colecistectomía & 588 & \multirow[t]{11}{*}{1.051} & \multirow[t]{11}{*}{302} & \multirow[t]{11}{*}{172} & \multirow{11}{*}{$\begin{array}{l}\text { Mycobac- } \\
\text { terium } \\
\text { masiliense }\end{array}$} & \multirow{11}{*}{$\begin{array}{l}15-30 \mathrm{mi} \\
\text { nutos }\end{array}$} \\
\hline & $\begin{array}{l}\text { Laparoscopia } \\
\text { diagnóstica }\end{array}$ & 82 & & & & & \\
\hline & Tubectomía & 24 & & & & & \\
\hline \multirow{8}{*}{$\begin{array}{l}\text { Duarte y } \\
\text { colaborado- } \\
\text { res [43] }\end{array}$} & Miomectomía & 24 & & & & & \\
\hline & Ooforectomía & 33 & & & & & \\
\hline & Hernioplastia & 24 & & & & & \\
\hline & Apendicectomía & 75 & & & & & \\
\hline & Artroscopia & 58 & & & & & \\
\hline & Gastroplastia & 16 & & & & & \\
\hline & $\begin{array}{l}\text { Rectosigmoi- } \\
\text { dectomía }\end{array}$ & 16 & & & & & \\
\hline & $\begin{array}{l}\text { Cirugía lapa- } \\
\text { roscópica no } \\
\text { especificada }\end{array}$ & 111 & & & & & \\
\hline
\end{tabular}




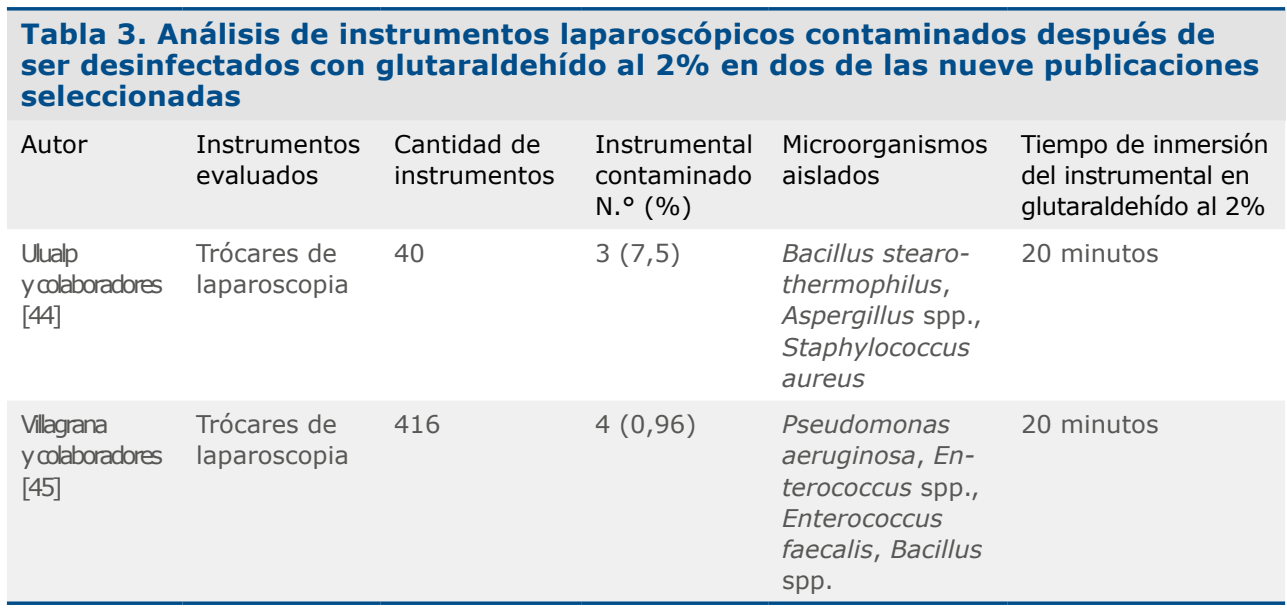

\section{Discusión}

La infección de sitio quirúrgico es un fenómeno asociado a las alteraciones en la ejecución de alguno de los procesos críticos de un procedimiento quirúrgico, bien sea en la atención directa del paciente, debido a la transgresión en la aplicación de las prácticas de control de infecciones cuya consecuencia principal es la ruptura de la barrera epidemiológica de transmisión de patógenos [35, 36, 46, 47], o en la preparación del material médico-quirúrgico que se empleará durante el procedimiento. Este panorama representa un número considerable de variables independientes una de la otra, que siempre han de ser tenidas en cuenta para obtener resultados satisfactorios en la atención del paciente, en este caso en el ámbito quirúrgico [26].

En la literatura médica se ha descrito la importante conexión que existe entre las infecciones del sitio quirúrgico y la incorrecta preparación antiséptica de la piel o las membranas mucosas del paciente en el área donde va a ser llevada a cabo la intervención [46]. La mayor parte de los microorganismos aislados e identificados mediante cultivos a partir del instrumental utilizado durante la cirugía, una vez terminada, constituyen elementos de la microbiota residente de la piel o las cavidades no estériles del ser humano $[40,48]$. Por otro lado, la contaminación cruzada de la herida quirúrgica con microorganismos provenientes de piezas anatómicas resecadas que entran en contacto directo con las superficies cruentas, como los puertos de laparoscopia, ha sido confirmada como un factor más fuertemente asociado a la infección posoperatoria en el sitio quirúrgico [46].

La capacitación del personal que manipula las unidades de esterilización en baja temperatura, requerida por el delicado instrumental laparoscópico, la infraestructura adecuada que según las reglamentaciones regionales deben tener las instalaciones donde operan, así como el manejo especial de los desechos que estos 
procesos generan y el tiempo prolongado que se requiere para proporcionar resultados efectivos en la eliminación de microorganismos patógenos, son componentes que hacen que la esterilización a baja temperatura sea una alternativa costosa, especialmente teniendo en cuenta que la cantidad de instrumental requerido para abastecer el número cada vez más creciente de intervenciones por mínimo acceso es limitado en la mayor parte de hospitales [24, 27]. Estas razones han constituido un argumento suficiente para que las instituciones de salud recurran a prácticas menos costosas como la desinfección de alto nivel.

Varios artículos ponen de manifiesto la seguridad que representa la desinfección de alto nivel con solución alcalinizada de glutaraldehído al $2 \%$ de los dispositivos de acceso e instrumental que será utilizado durante la ejecución de diversos tipos de cirugías por vía mínimamente invasiva, siempre y cuando dicho proceso sea llevado a cabo de manera rigurosa, siguiendo los protocolos previamente establecidos por los entes de control que aseguran su eficacia y por el personal debidamente capacitado para ello $[49,50]$.

La inadecuada dilución del desinfectante, los tiempos de inmersión insuficientes, el uso del desinfectante luego de que su período de actividad haya caducado, así como la manipulación incorrecta por personal sin instrucción, son, entre otros, factores que alteran la eficacia del proceso de desinfección de alto nivel. Cualquier falla presentada durante el proceso de desinfección resulta en una reducción de la eficacia del mismo, cuya consecuencia directa es la contaminación microbiana de los dispositivos médico-quirúrgicos, que posteriormente deriva en una infección ya sea en la herida operatoria o en las cavidades del paciente $[6,27,33,51]$.

A pesar de que el glutaraldehído al 2\%, como desinfectante de alto nivel, presenta una amplia actividad microbicida demostrada sobre 20 tipos de microorganismos diferentes, incluyendo bacterias en estado vegetativo, micobacterias, hongos y virus $[6,39,40]$, las infecciones descritas en esta revisión, causadas principalmente por microorganismos atípicos como Mycobacterium masiliense, el cual presenta una tolerancia comprobada a las soluciones comerciales a base de glutaraldehído al $2 \%$, producto más frecuentemente utilizado para realizar la desinfección de alto nivel, representan un nuevo reto en cuanto a la seguridad del paciente y la efectividad de los procesos de desinfección que deben aplicar las instituciones de salud para prevenir las infecciones por este tipo de microorganismos. Mycobacterium masiliense puede ser aislado tras períodos de exposición al glutaraldehído al $2 \%$ tan largos como 10 horas $[42,43,52,53]$. Además, la colonización demostrada en los trócares laparóscopicos de reúso establece un riesgo potencial de infección que va a depender de la cantidad de unidades formadoras de colonias o biopelículas en el instrumental y el estado inmunológico del paciente. En conjunto, estos hallazgos representan una de las mayores preocupaciones actuales en la comunidad médica en la decisión de desinfectar el instrumental quirúrgico y cómo hacerlo adecuadamente o, en su lugar, someterlo a un proceso de esterilización. 


\section{Conclusiones}

Se evidencia que las condiciones de almacenamiento, preparación, limpieza y dilución del glutaraldehído al 2\%, al igual que el manejo y la capacitación que el personal tiene sobre esta solución, están asociadas a la falta de efectividad del proceso de desinfección de alto nivel del instrumental laparoscópico, lo que lleva a reevaluar su uso en las instituciones de salud. Se destaca la implicación de microorganismos atípicos como Mycobacterium masiliense como agentes causales de infección del sitio quirúrgico debido al uso de material laparóscopico desinfectado con glutaraldehído al $2 \%$.

La decisión de desinfectar el instrumental laparoscópico mediante glutaraldehído al $2 \%$, en lugar de someterlo a un proceso de esterilización, lleva a una disminución considerable en los costos de los hospitales; sin embargo, dicho tratamiento, cuando realizado bajo condiciones no óptimas o por personal no entrenado, supone someter a los pacientes a un mayor riesgo de contraer una infección del sitio quirúrgico gracias al contacto de los tejidos vivos con instrumental potencialmente contaminado.

La esterilización, como el mecanismo más efectivo para la eliminación de microorganismos, siempre que sea posible, debe ser el método de elección para el tratamiento de los instrumentos quirúrgicos. El profesional encargado de la central de esterilización debe valorar las potenciales ventajas de la desinfección de alto nivel, siempre y cuando se respeten las recomendaciones ofrecidas por los fabricantes tanto para el instrumental quirúrgico como para las sustancias desinfectantes, así como mantener siempre la rigurosidad en cuanto a las condiciones sanitarias necesarias y la capacitación del personal delegado para realizar la desinfección de dicho instrumental.

\section{Bibliografía}

1. Solano V, Solano R. Colecistectomía laparoscópica por puerto umbilical asistido. Rev Chil Cir 2012; 64: 147-154.

2. Pérez-Albacete $\mathbf{M}$. Historia de la cirugía laparoscópica y de la terapia minimamente invasiva. Clín Urol Complut 2005; 11: 15-44.

3. Camacho FJ, Ramírez JF, Herrera DP, Cortés M. Curso básico de entrenamiento en habilidades para cirugía endoscopica: grado de satisfaccion de los profesionales en formacion. Rev Col Or Tra 2009; 23: 16-20.

4. Fernández-Santiesteban $L$, Silvera-García JR, Díaz-Calderín JM, Vilorio-Haza P, Loys-Fernández JL. Cirugía videolaparoscópica en urgencias. Rev Cubana Cir 2003; 42.

5. Bernabéu A. Tratamiento de las cicatrices.
Rev Offarm 2009; 28: 38-41.

6. Rutala WA, Weber DJ, Healthcare Infection Control Practices Advisory Committee (HICPAC). 2008. Guidelines for disinfection and sterilization in healthcare facilities. http://www.cdc.gov/hicpac/pdf/guidelines/ Disinfection_Nov_2008.pdf, accessed marzo 2013.

7. Ercole FF, Chianca TCM, Duarte D, Starling CEF, Carneiro M. Surgical site infection in patients submitted to orthopedic surgery: the NNIS risk index and risk prediction. Rev Latino-Am Enfermagem 2011; 19: 269-276.

8. Wenzel RP. Health care-associated infections: major issues in the early years of the 21st century. Clin Infect Dis 2007; 45: S85S88. 
9. Cuellar L. Infecciones nosocomiales. Rev Diagnóstico 2009; 48: 13-15.

10. Silvestre C, Fagoaga L, Garciandía MJ, Lanzeta I, Mateo MC, Zapata, M.C. Esterilización. ANALES Sis San Navarra 2000; 23: 95-103.

11. Rosa AC, González MI. Agentes físicos para el control de microorganismos. In: Negroni M, ed. Microbiología Estomatológica: Fundamentos y guía práctica (ed 2da). Buenos Aires, Argentina: Editorial Médica Paramericana; 2009: 133-148.

12. Friedman C, Newsom W. Conceptos básicos de control de infecciones de IFIC (ed 2da). Irlanda-Gran Bretaña: International Federation of Infection Control; 2011.

13. Goveia VR, Pinheiro SMC, Graziano KU. Métodos de esterilização por baixa-temperatura e novas tecnologias. Rev Latino-am Enfermagem 2007; 15: 373-377.

14. Balagué C, Targarona EM, Trias M. Cirugía laparoscópica e infección quirúrgica. Rev Esp de Cir 2000; 67: 184-191.

15. Lopes CdLBC, Graziano KU, Pinto TdJA. Evaluation of single-use reprocessed laparoscopic instrument sterilization. Rev Latino-Am Enfermagem 2011; 19: 370-377.

16. Quiñones $\mathbf{M}$. Cuidados y mantención del instrumental laparoscópico. Medwave 2005; 5: e2682.

17. Hidalgo-Rodríguez $\mathbf{R}, \quad$ CastellanosFernández VM, Chiroles-Despaigne $S$, Villavicencio-Betancourt $\boldsymbol{O}$. Dispositivos médicos de uso único reprocesados por esterilización química mediante óxido de etileno. Rev Cubana Hig Epidemiol 2002; 40: 89-94.

18. Glaser ZR. El futuro del óxido de etileno como esterilizante para dispositivos médicos. El Autoclave (Revista del Club Español de Estirilización) 2002; 14: 37-40.

19. Barrera Barroso L, Otero Abreu I, Rodríguez Nápoles D, González Rodríguez $Y$. Empleo de las radiaciones gamma como método de esterilización en biomateriales. Revista CENIC Ciencias Químicas 2005; 36: 1-5.

20. Rincón A AC, Herrero V R, Rodríguez C J, Alonso $\mathbf{M}$, Molina $\mathbf{I}$. Influencia de la radiación gamma como método de esterilización sobre el polímero poli VPAVG (Valina-ProlinaAlanina-Valina- Glicina). INHRR 2009; 40: 26-35.

21. Hernández-Martín JM. Nueva generación de esterilizadores por gas plasma H2O2 STERRAD $尺$ NX. El Autoclave (Revista del Club Español de Estirilización) 2005; 17: 7-13.

22. Lama-Ochoa de Retama MA. Limpieza y esterilización en quirófano de oftalomología.
Tesis para optar al título de Máster en Enfermería Oftalmológica. Bilbao, España: Universidad de Valladolid. Escuela Universitaria de Enfermería; 2012.

23. Acosta-Gnass SI, de Andrade Stempliuk V. 2008. Manual de esterilización para centros de salud. http://www.paho.org/PAHO-USAID/ dmdocuments/AMR-Manual_Esterilizacion_ Centros_Salud_2008.pdf, accessed mayo 2013.

24. Rutala WA, Weber DJ. New disinfection and sterilization methods. Emerg Infect Dis 2001; 7: 348-353.

25. Albornoz H, Guerra S. 2008. Manual de prevención de infecciones en procedimientos endoscópicos. http://www.cocemi.com.uy/ docs/endo2008.pdf, accessed mayo 2013.

26. Rodríguez Pérez AU. La desinfección-antisepsia y esterilización en instituciones de salud. Atención primaria. Rev Cubana Med Gen Integr 2006; 22: 1-11.

27. Nachón FJ, Díaz J, Rivas V, González JS, Nachón MG, García F, et al. Esterilización por inmersión de instrumental de cirugía laparoscópica. Estudio comparativo entre glutaraldehído al 2\%, agua electrolizada superoxidada con $\mathrm{pH}$ neutro y solución electrolizada por selectividad iónica con $\mathrm{pH}$ neutro. Rev Med UV 2008; 8: 5-10.

28. Gómez E. Desinfección semi-automática de endoscopios: Stella \& Pulse. El Autoclave (Revista del Club Español de Estirilización) 2011; 23: 4-8.

29. Muro-Ceballos I. Esterilización en punto de uso. El Autoclave (Revista del Club Español de Estirilización) 2003; 15: 20-23.

30. Sallés M, Codina C. 2005. Higiene y antisepsia del paciente. Limpieza, desinfección y esterilización en el ámbito hospitalario. http:// www.scfarmclin.org/docs/higiene/higiene. pdf, accessed mayo 2013.

31. Lorduy G, Carrillo S. Contaminación de desinfectantes usados en el área quirúrgica ¿Potencial bacteriano intrínseco, resistencia adquirida o factores extrínsecos relacionados? Rev Cienc Salud Virtual 2011; 3: 144-153.

32. Manzoor SE, Lambert PA, Griffiths PA, Gill MJ, Fraise AP. Reduced glutaraldehyde susceptibility in Mycobacterium chelonae associated with altered cell wall polysaccharides. J Antimicrob Chemother 1999; 43: 759-765.

33. Vignoli G. Esterilización, desinfección y antisepsia. Temas de Bacteriología y Virología Médica. Montevideo, Uruguay: Departamento de Bacteriología y Virología, Instituto de Higiene, Universidad de La Republica; 2008: 609-629.

34. Álvarez-Hernández G, Amaro-Ortega C. 
Costos atribuibles y factores de riesgo de infección nosocomial en un hospital del Estado de Sonor. Bol Med Hosp Infant Mex 2010; 67: 118-127.

35. Rodas JH, Ruiz Pérez VE, Villalba J. Infección intrahospitalaria en cirugía electiva: frecuencia y costo. Rev Chilena de Cirugía 2002; 54: $362-367$

36. Arévalo H, Cruz R, Palomino F, Fernández F, Guzmán E, Melgar R. Aplicación de un programa de control de infecciones intrahospitalarias en establecimientos de salud de la región San Martín, Perú. Rev Peru Med Exp Salud Publica 2003; 20: 84-91.

37. Zayyan KS, Sellu DP. High-level disinfection with $2 \%$ alkalinized glutaraldehyde solution for reuse of laparoscopic disposable plastic trocars. J Laparoendosc Adv Surg Tech A 1999; 9: 117-118.

38. Rodrigues C, Mehta A, Jha U, Bharucha M, Dastur FD, Udwadia TE. Nosocomial Mycobacterium chelonae infection in laparoscopic surgery. Infect Control Hosp Epidemiol 2001; 22: 474-475.

39. Ramesh H, Prakash $K$, Lekha V, Jacob G, Venugopal A, Venugopal B. Port-site tuberculosis after laparoscopy: report of eight cases. Surg Endosc 2003; 17: 930-932.

40. Colak T, Ersoz G, Akca T, Kanik A, Aydin S. Efficacy and safety of reuse of disposable laparoscopic instruments in laparoscopic cholecystectomy: a prospective randomized study. Surg Endosc 2004; 18: 727-731.

41. Vijayaraghavan $\mathbf{R}$, Chandrashekhar $\mathbf{R}$, Sujatha Y, Belagavi CS. Hospital outbreak of atypical mycobacterial infection of port sites after laparoscopic surgery. J Hosp Infect 2006; 64: 344-347.

42. Lorena NS, Duarte RS, Pitombo MB. [Rapidly growing mycobacteria infection after videosurgical procedures - the glutaraldehyde hypothesis]. Rev Col Bras Cir 2009; 36: 266267.

43. Duarte RS, Lourenco MC, Fonseca Lde S, Leao SC, Amorim Ede L, Rocha IL, et al. Epidemic of postsurgical infections caused by Mycobacterium massiliense. J Clin Microbiol 2009; 47: 2149-2155.
44. Ulualp KM, Hamzaoglu I, Ulgen SK, Sahin DA, Saribas S, Ozturk $\mathbf{R}$, et al. Is it possible to resterilize disposable laparoscopy trocars in a hospital setting? Surg Laparosc Endosc Percutan Tech 2000; 10: 59-62.

45. Villagrana J, Solano R, Ibarra V, Sosa $I_{\text {, }}$ Quesnel C, Ahued J. Análisis microbiológico de la reutilización de trócares de laparoscopia en un hospital de tercer nivel. Perinatol Reprod Hum 2002; 16: 96-202.

46. Mangram AJ, Horan TC, Pearson ML, Silver LC, Jarvis WR. Guideline for prevention of surgical site infection, 1999. Hospital Infection Control Practices Advisory Committee. Infect Control Hosp Epidemiol 1999; 20: 250278.

47. Nodarse-Hernández R. Visión actualizada de las infecciones intrahospitalarias. Rev Cub Med Mil 2002; 31: 201-208.

48. Loffer FD. Disinfection vs. sterilization of gynecologic laparoscopy equipment. The experience of the Phoenix Surgicenter. J Reprod Med 1980; 25: 263-266.

49. Gundogdu H, Ocal K, Caglikulekci $\mathbf{M}$, Karabiber N, Bayramoglu E, Karahan $\mathbf{M}$. High-level disinfection with $2 \%$ alkalinized glutaraldehyde solution for reuse of laparoscopic disposable plastic trocars. J Laparoendosc Adv Surg Tech A 1998; 8: 47-52.

50. Dumartin C, Brucker G. [Rules of decontamination and disinfection of medicosurgical instruments in the operating room]. Ann Chir 1995; 49: 173-179.

51. Prieto-de Lamo G, Rey-Liste MT. 2005. Efectividad y seguridad del orto-ftalaldehído en la desinfección de alto nivel de material sanitario. http://www.sergas.es/cas/Servicios/docs/AvaliacionTecnoloxias/OPA\%20 CT2005_02.pdf, accessed mayo 2013.

52. Sabagh BP, Souto Ada S, Reis LM, Silva SA, Pereira DC, Neves Mde C, et al. Comparative study with two different enrichments in the culture media used in the disinfectant efficacy assay. J Microbiol Methods 2012; 88: 255-262.

53. Roth K, Heeg P, Reichl R. Specific hygiene issues relating to reprocessing and reuse of single-use devices for laparoscopic surgery. Surg Endosc 2002; 16: 1091-1097. 